Ryan, A. A.; Letchford, K. A.; Senge, M. O. (2014):

Tailoring Porphyrin (Nano)structures.

ECS Transactions 61, 1-12.

\title{
Tailoring Porphyrin (Nano)structures
}

\author{
A. A. Ryan ${ }^{\mathrm{a}}$, K. A. Letchford ${ }^{\mathrm{a}}$ and M. O. Senge $\mathrm{e}^{\mathrm{a},}$ \\ ${ }^{a}$ SFI Tetrapyrrole Laboratory, School of Chemistry, Trinity Biomedical Sciences \\ Institute, 152-160 Pearse St., Trinity College Dublin, The University of Dublin, Dublin 2, \\ Ireland
}

\begin{abstract}
Porphyrins present one of the most ubiquitous classes of compounds in chemistry, optics, medicine, and materials science. This is a result of their multifaceted roles in nature and the wide range of tunable molecular properties. Further advances in translational research and a more detailed understanding of their biological function require a specific tailoring of the macrocycle to the desired task. Using examples from our own research we illustrate how a targeted molecular design combining the aspects of conformational flexibility with rational chemical synthesis in porphyrins can be used to advance our knowledge of cofactor function and to develop novel and highly efficient materials.
\end{abstract}

\section{Introduction}

Porphyrin chemistry is a rapidly expanding field, today mainly driven by advances in materials sciences and biological applications (1). Porphyrins are an essential class of pigments that are found widespread across nature throughout various biological systems. Many synthetic derivatives are continuously being developed (2) to increase and improve their use in many different applications including medicine, optical agents, and photovoltaics (3-6).

In chemical terms the porphyrin macrocycle $\mathbf{1}$ can act as a functionalization scaffold to design useful compounds. In practice, there are several variable positions at which the macrocycle and core structures of porphyrins can be altered or manipulated by chemical means. Variable positions include meso-substitutions i.e., the introduction of sterically demanding residues, transmetallation, substitutions of axial ligands, reductions, disruption of the $\pi$-conjugated system, $N$-substitution, cation radical formation, covalent linkage at $\beta$ or meso-positions by other compounds, and lastly, heteroatom substitutions $(1,2)$. Variations of most types can alter the conformation of the porphyrin and the overall physicochemical properties of the compound.

In applications, meso-substituted porphyrins are arguable the most the most versatile kind of synthetically modified structures. They can be symmetrical, - i.e., the classic 5,10,15,20-tetraarylporphyrins - however, most modern applications employ unsymmetrically substituted porphyrins, derived from either $A_{x}$ (only one type of mesosubstituent) or ABCD-type systems (4, four different meso-substituents). Judicious choice of such substituents can affect the physicochemical properties of parent macrocycles in such a way that they can be tailored to suit different applications (Figure 1) (2). 


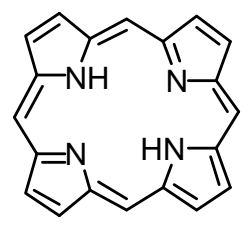

1 porphyrin

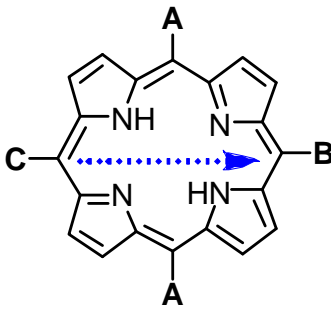

$2 \mathrm{~A}_{2} \mathrm{BC}$ porphyrin

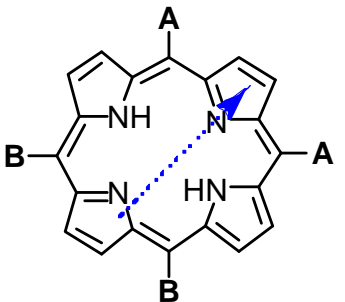

$3 \quad A_{2} B_{2}$ porphyrin

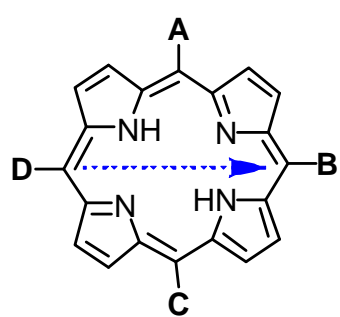

4 ABCD porphyrin

indicates molecular dipole moments

Figure 1. Basic molecular substituent types of porphyrins.

Their highly conjugated aromatic macrocyclic system and well-understood structural and physical chemistry are features that make porphyrins valuable in optical applications. Properties such as solubility, optical clarity, thermal stability, and absorption can be controlled through specific molecular design, allowing for them to have features specific to different applications (3b). As optical materials, they have a rich photochemistry with features such as non-linearity, fast response times, broadband spectral results, and longlived triplet states/lifetimes. These features favor their use as optical materials in fields such as telecommunications, displays, data storage and photomedicine.

Likewise, porphyrins are employed as molecular semi-conductors in photovoltaic cells for photoelectronic applications. Large extinction coefficients, low processing costs, and a wide scope of possibilities for synthetic variations are features that make them valuable materials for these processes (4). Synthetic model systems are used to mimic the effect of electron-transfer reactions in light-harvesting complexes involving chromophores. Porphyrin arrays can be designed and synthesized to act as lightharvesting devices in which photonic energy is converted into electronic transfer and electrical energy. Conjugation within these arrays enable long range energy transfer and high electronic mobility through electron hopping, ground- to excited- state excitation, and spin exchange between the conjugated units (7).

Porphyrins are also used as photosensitizing (PS) agents in photodynamic therapy (PDT). PDT uses light initiated reactions for the treatment of many diseases including cancer, psoriasis, age-related macular degeneration (AMD), and arthritis (5). Firstly, the photosensitizing agent is administered; ideally it is non-toxic and selectively accumulative in target tissues. In the presence of light of a suitable wavelength, the molecules will absorb a photon of light. The photosensitizer molecule will undergo promotion from a ground- to a singlet excited-state. The PS agent will become active by intersystem crossing to form a triplet excited-state. Energy transfer to molecular oxygen will form highly reactive, singlet oxygen species that can induce aptosis or necrosis in cancerous cells.

Porphyrins serve many important functions in nature. Pigment-protein complexes with porphyrin cofactors catalyze reactions in biological systems that are crucial to life. In photosynthesis, photons of light are absorbed resulting in electronic excitation and a chain of electron transfer reactions from light harvesting chlorophyll to a reaction centre. 
The highly conjugated $\pi$-system of porphyrins and their spatial arrangement drive and enable the long-distance electron transfer processes. These events lead to a charge separation across a membrane and the potential energy can be used to generate large amounts of ATP. Efforts have been made to develop biomimetic systems for this process such that solar excitation energy can be converted into potential energy and then electrical energy. Here, many efforts are being made to develop synthetic porphyrin systems to act as models for the natural function of chlorophylls (7a).

The development of novel optically active nano- and micro-materials requires access to appropriate molecular materials. Especially in the area of nanomaterials this necessitates that the basic molecular materials are optimised for their applications on the molecular level. Optimisation of optical properties of the active medium can not only be achieved in materials applications but has to be "imprinted" in the individual molecules. This can only be done when appropriate synthetic methods are available to prepare molecules in high yields and with high selectivity, and to fine-tune the physicochemical properties of the parent compounds. Naturally, this has to go hand in hand with a detailed understanding of the molecular features that govern the photoexcited properties of the chromophores, which is necessary to identify key molecular features to be included in the optimised target compounds.

In a conceptual sense, the main question is how to impart the desired physicochemical properties into the molecules and whether this can be done in a general way or requires different strategies for each application area? Some approaches, such as the modulation of the chromophore through introduction of electron donating or withdrawing groups, the varying effect of the central metal, or the replacement of macrocycle atoms with heteroatoms are straightforward. Similarly, the development of 'fusing'-reactions for the generation of $\pi$-extended porphyrinoids is progressing rapidly. Others, e.g., the effects of surface deposition of porphyrins or noncovalent $\pi-\pi$ interactions are still in its infancy and/or difficult to control for translational research. Finally, the primary mode of how nature controls the function of porphyrin cofactors - through spatial and conformational control - is only slowly making its impact in applied porphyrin research. Here we give a brief account of our own answer(s) to this question using organic chemistry, surface studies and an investigation of biological systems.

\section{Organic Synthesis}

\section{Unsymmetrical ABCD-type Porphyrins}

By now the synthetic organic chemistry of porphyrins is well developed and has expanded into almost any facet of cyclic, aromatic oligopyrrole derivatives (9). A typical example of recent advances in the core subject is the possibility to prepare unsymmetrically meso substituted porphyrins (ABCD-type systems) $(2,10)$. In principle, there are three methods by which these compounds can be synthesized. Firstly is through mixed condensations whereby one pyrrole-containing molecule is condensed with two or more aldehydes. Obtaining the target compound is problematic and not always successful as tedious chromatographic work-up is required to separate the target compound from the mixture of isomeric products. The second method of production is by total synthesis synthetically this is the most logical route but problems arise as this method involves many synthetic steps and the possibility of acid-catalyzed scrambling during the 
oxidation step could lower the yield of the target compound. Thus, contemporary methods utilize the functionalization of preformed systems - this is our main approach. It involves the preparation of a mono-substituted A-type porphyrin followed by a series of functionalization reactions involving the sequential regiochemical introduction of B-, C-, and D-type residues $(2,10 \mathrm{~b})$.

Once the necessary starting materials are available, there are three key reactions employed throughout functionalization sequences for the conversion from $\mathrm{A}-\rightarrow \mathrm{AB} \rightarrow$ ABC- $\rightarrow$ ABCD-type porphyrins. These are: (a) mono-substitutions by sequential reactions with organolithium reagents (9c), (b) one-pot reactions, resulting in disubstitution at two meso-positions, and (c) the use of transition metal (mainly Pd) catalyzed C-C coupling reactions (11). Generally a combination of the different synthetic strategies is used to optimize yields. There is no generally preferred sequence for the synthesis of different porphyrins, each target compound has its own synthetic route depending on different factors including the substituents involved $(2,10 \mathrm{a})$.

Mono-substitutions using organolithium (RLi) reagents employ $\mathrm{S}_{N} \mathrm{Ar}$ reactions where the RLi reagents are prepared using various synthetic methods and are reacted with metallo- or free base porphyrins (12). The method can be repeated for each free meso position. However, the availability of RLi reagents could be limited and the use of sterically hindered reagents, such as ${ }^{t} \mathrm{BuLi}$ may often result in multiple alkylation and/or $\beta$-alkylation reactions. RLi reagents can be used for further functionalization of fullymeso substituted porphyrins, i.e., in the preparation of phlorins, porphodimethenes $(5,15-$ dihydroporphyrins), or chlorins (12). One-pot reactions are derived from the previous method. They involve the in situ trapping of the anionic intermediate from the nucleophilic attack of $\mathrm{RLi}$ on the porphyrin species. The addition of an organic electrophile will lead to a disubstituted product - one residue derived from RLi and the other from the electrophile (12d). Therefore, in this method, the conversion of an ABtype porphyrin to an ABCD-type is possible. Lastly, the most widely used methods use a range of Pd-catalyzed reactions - mainly Heck, Suzuki, Sonogashira, and Stille - in the production of ABCD-type compounds. Firstly, bromination or iodination of a free mesoposition is performed followed by a C-C coupling reaction. Yields are generally good to excellent; however, variations depend on the individual porphyrin and coupling partners employed in the reaction. Limitations exist with the introduction of heteroatom-linked alkyl residues and in forming regioselective products by the halogenation of porphyrins with more than one free meso-position. Overall, by using a combination of these three reaction classes in step-wise sequences it is possible to obtain almost any ABCDporphyrin, in theory at least $(2,10)$.

Such meso substitutions can be used for the electronic modulation of macrocycles through the variation of different functional groups. Classic examples are the push-pull porphyrins (13). Systems such as these carry an electron-donating group and an electronwithdrawing group on opposite sides of the macrocycle (3b). They can exhibit charge transfer character and are highly studied as biomimetic electron transfer compounds. Push-pull systems are of great use in non-linear optical (NLO) applications. NLO devices require asymmetric molecules with strong intramolecular, permanent dipole moments. Therefore, the insertion of suitable donating/acceptor meso-substituents using the above methods can improve NLO responses and allow for fine-tuning of optical properties within such devices (3b). 
Unsymmetrical $\mathrm{A}_{2} \mathrm{BC}$-type porphyrins 2 are of the classic push-pull type in which there is electron donating and an accepting substituent with a 5,15-disubstitution pattern. In principle, by increasing the strength of the intramolecular dipole moment of the molecule, the NLO response can be optimized. A new series, the 5,10- $\mathrm{A}_{2} \mathrm{~B}_{2}$-type 3 , have been developed by us in which the intramolecular dipole is strengthened by modulation of the excited state properties (14a). Properties are also varied by conformational design, molecular symmetry, and as in this case - changes in donor and acceptor substituents. This class has two donor and two acceptor groups added, respectively at meso-positions of the $\pi$-system, i.e. in a 5,10-disubstitution pattern.

Non-linear absorption (NLA) properties of free-base and metallated 5,10- $\mathrm{A}_{2} \mathrm{~B}_{2}$ substituted porphyrin series' were analyzed to determine their optical properties relative to those with different substitution patterns. These systems are an interesting class of porphyrin compounds due to their unique alignment and the strength of their intramolecular dipole moments (14). A series of models were considered to describe the evolution of NLA with the input fluence. The responses received were diverse showing RSA/SA, RSA/RSA, and SA/RSA switches depending on the structural features, i.e., the metal center, of the compounds. A 4-level model with simultaneous 2-photon absorption arising from the higher excited state of the compound (consecutive $1+1+2$ photon absorption) was successfully fitted to these 5,10- $\mathrm{A}_{2} \mathrm{~B}_{2}$-compounds, including those in different solvents. The model indicated that different $5,10-\mathrm{A}_{2} \mathrm{~B}_{2}$-compounds possess similar excited state character and behavior (12b). The regiochemical substitution pattern lead to NLA properties unique to other fully meso-substituted compounds including 5,15$\mathrm{A}_{2} \mathrm{~B}_{2} / 5,15-\mathrm{A}_{2} \mathrm{BC}$-type systems due to differences in their excited state structures. In optical limiting devices, compounds will ideally exhibit a drop in transmission with increasing input fluence due to the evolution of higher excited states, as demonstrated by $5,10-\mathrm{A}_{2} \mathrm{~B}_{2}$-compounds. These compounds exhibit higher quantum yields, faster intersystem crossing, and higher ratios of excited state to ground state absorption crosssections. Porphyrin-solvent interactions were shown to have an effect on the NLA properties of the compounds. Solvent types can alter ground state and excited state properties in compounds and therefore, affect their potential as optical limiting agents. They can alter the magnitude of the drop or increase in transmission and also the RSA/SA or SA/RSA switch fluence $(3 \mathrm{c}, 12 \mathrm{c})$.

\section{Extension of the Aromatic System}

Many medical and optical applications require the use of far-red absorbing dyes. Due to this, we have developed methods to synthesize porphyrin dimers (Figure 2). Our group focused on the synthesis of triply-fused bisporphyrins 6 in which conjugation in the systems is maximized and can be extended to form arrays. One route involved oxidative fusing reactions of symmetric and/or unsymmetric dimers such as 5. Another more attractive route involved a one-electron chemical oxidation of monomeric zinc(II) porphyrins with a free-meso position 7 to yield symmetric products. Extension of the $\pi$ conjugated in these arrays causes dramatic bathochromic shifts in the absorption spectrum into the near-IR region. Shifts can be enhanced further to absorptions at $>1050$ $\mathrm{nm}$ by direct functionalization reactions that enable the fine-tuning of these arrays to suit different applications. Such bisporphyrins can be used in application such as NLO (3b), organic light-emitting diodes (OLEDs) (15), and two-photon absorption PDT $(16,17)$. 


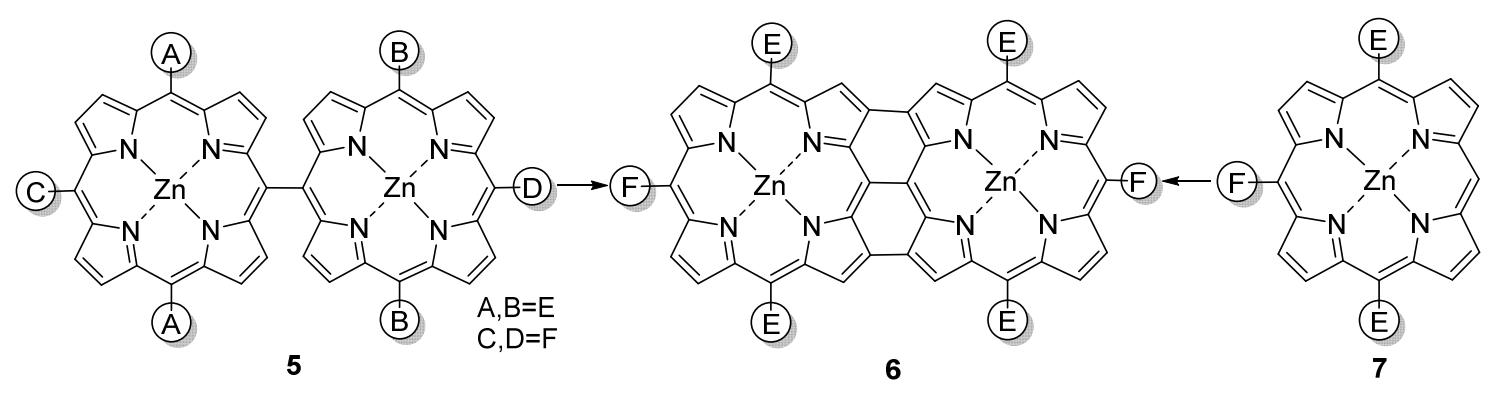

Figure 2. Synthesis of triply fused dimers 6 via symmetric and unsymmetric directlylinked zinc(II) porphyrin dimers (5) and 5,10,15-trisubstituted zinc(II) porphyrin precursors (7).

Symmetric directly linked free base bisporphyrins and their $\mathrm{Zn}(\mathrm{II})$ derivatives such as 5 were obtained by PIFA oxidation and organolithium reactions from 5,15-disubstituted porphyrins in good yields (59-74\%) (18). Monomeric porphyrins with electronegative substituents, i.e. 5-bromo-10,20-disubstituted porphyrins, produced dimers in good-toexcellent yields (58 and 86\%). Dimers with two free meso positions are desirable for further functionalization purposes; however, isolating them using this method was unsuccessful due to the formation of oligomerized products. Use of PIFA or $\mathrm{DDQ} / \mathrm{Sc}(\mathrm{OTf})_{3}$ in excess allowed the synthesis of triply fused $\beta-\beta$, meso-meso, and $\beta$ ' $-\beta$ ' linked bisporphyrins $6(19,20)$.

Another successful method utilized a stepwise synthetic strategy incorporating Suzuki-Miyaura couplings. Here, coupling between bromoporphyrins and borylated porphyrins (21) yielded meso-meso linked porphyrin dimers in good yields (43-66\%). However, homocoupling between borylated porphyrins generated unwanted dimeric side products (22). The resulting bisporphyrin could undergo further reactions to triply-fused systems and/or functionalization reactions could be performed at free-meso positions (20). Note, that various studies have been reported in this area, many of them going back to Osuka's development of the triply-fused systems (10a,22,23,24,25,26). Typically, zinc(II) porphyrins fare better in these reactions as they are more easily oxidized than $\mathrm{Ni}$ or Pd, therefore aiding the oxidation process (27).

Triply-fused unsymmetric dimers were synthesized using a similar strategy. First, directly singly-linked unsymmetric dimers were prepared and then fused oxidatively using $\mathrm{DDQ} / \mathrm{Sc}(\mathrm{OTf})_{3}$ or PIFA. Here, the results were mixed. With "meso free" bisporphyrins, fusing occurred at the unsubstituted meso position resulting in the formation of both dimeric and tetrameric products. For hexasubstituted dimers, good yields $(56 \%)$ of the triply-linked dimers were obtained, indicating that this method has further potential (20).

\section{$\underline{\text { Spatial Arrangement }}$}

Numerous examples for the logical construction of spatially well defined multiporphyrin architectures have been published over the years $(7 \mathrm{a}, 28)$. These relate to the highly ordered natural systems, such as multi-heme unit electron transfer systems, the photosynthetic reaction center with its special pair and accessory chlorophylls and 
pheophytins and the beautifully ordered light harvesting proteins with antenna chlorophylls (29). Synthetic chemists have thus used the whole repertoire of organic chemistry to construct 3D multiporphyrin architectures which mimic distance, orientation and $\pi$-overlap of natural pigment systems and study the impact of the spatial arrangement on their properties. Typical examples are cofacial porphyrins, conjugated oligoporphyrins and porphyrin-based nanostructures (30).
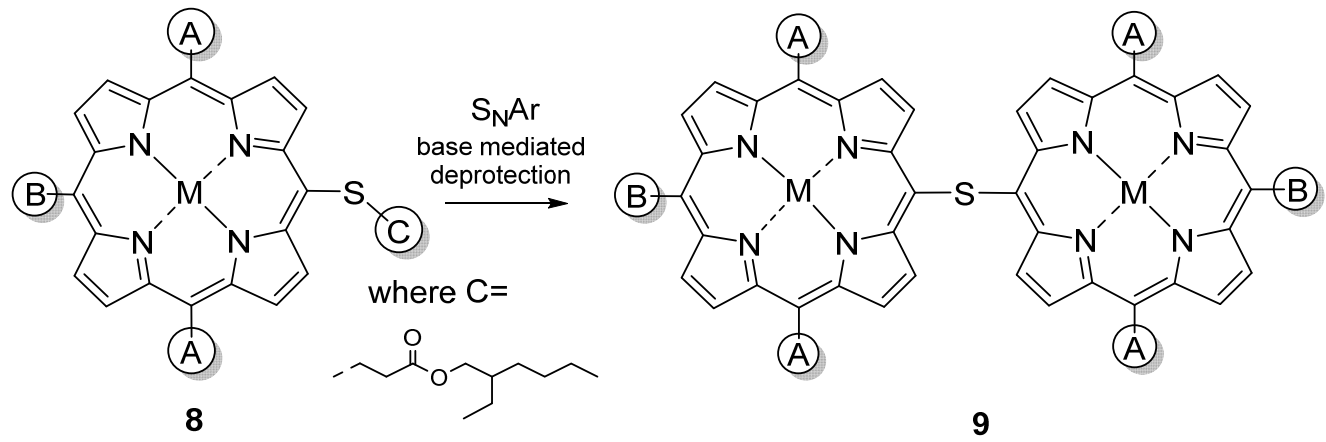

Figure 3. Synthesis of S-linked bisporphyrins 9 via base-mediated deprotection of thioether appended porphyrins 8.

A recent addition to the synthetic methodology for the generation of co-facial bisporphyrin structures was derived from our attempts to prepare thiol substituted porphyrins (31). Porphyrins bearing thiol groups have the ability to form self-assembled monolayers on gold surfaces making them a compound of interest in optical applications (32). To obtain such compounds, thioether masked porphyrins, such as porphyrin 8, were subjected to base-mediated deprotection (33). Unusual results were obtained with sulfurlinked bisporphyrins 9 isolated as the major product. A number of thioether substituted porphyrins were synthesized by a Pd-catalyzed reaction between bromoporphyrins and 2ethylhexyl-3-mercaptopropionate to generate porphyrins 8 in excellent yields (34). Upon base deprotection of the thioether chain, a thiolate anion was generated which underwent a nucleophilic aromatic substitution $\left(\mathrm{S}_{\mathrm{N}} \mathrm{Ar}\right)$ reaction in situ with $\mathbf{8}$ resulting in sulfurlinked porphyrin dimers 9 . All of the reactions went to completion with $\mathrm{Zn}$ (II) and $\mathrm{Ni}$ (II) dimers isolated in good-to-excellent yields of $55-72 \%$. The reaction was relatively simple to execute and prior to this, the synthesis of sulfur-linked bisporphyrins had not been reported. The excellent leaving group ability of isooctyl-3-mercaptopropionate as well as the strength of the thiolate porphyrin as a nucleophile drives the success of the reaction. Further insight into the addition/elimination mechanism involved was provided via displacement $\left(\mathrm{S}_{\mathrm{N}} \mathrm{Ar}\right)$ reactions with alkyl halides.

\section{Macrocycle Distortion}

Next to modulation via different functional groups or the preparation of oligomeric systems the simplest means to alter the physicochemical properties of porphyrins is through conformational distortion. As for any other planar aromatic system the introductions of bulky substituents or the promotion of peri-interactions may result in porphyrins with nonplanar macrocycles (Figure 4). As a result, the whole range of physicochemical parameters (redox potential, ground and excited state properties, absorption maxima, chemical reactivity, etc.) are altered in direct correlation with the 
degree of macrocycle distortion (35). This is now a well-established field and our recent contributions have focused on direct correlation of nonplanarity and photophysical parameters and the advancement of synthetic methods for conformationally distorted systems (36).
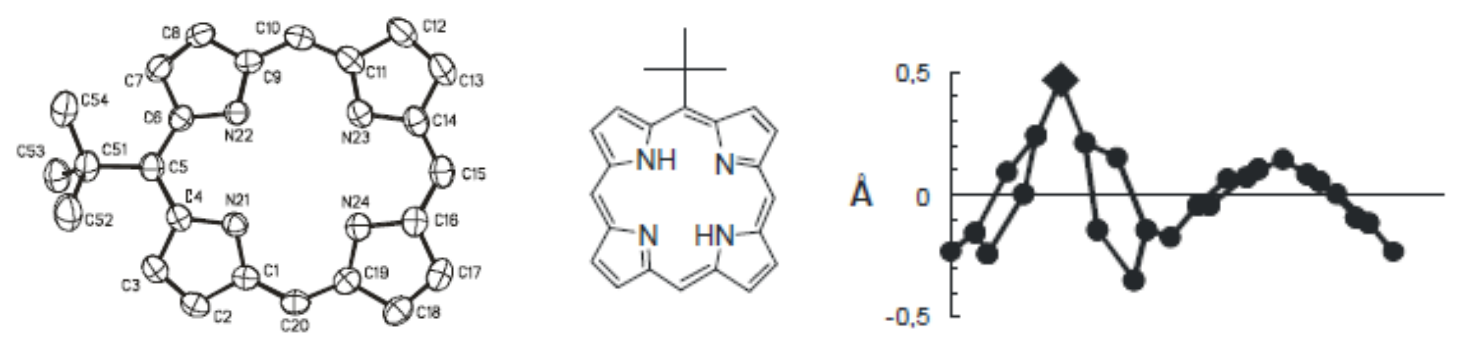

Figure 4. A typical nonplanar porphyrin: 5-tert-butylporphyrin (36e). From left to right: View of the molecular structure with hydrogen atoms omitted for clarity, molecular formula and view of the skeletal deviations of the macrocycle atoms from the $4 \mathrm{~N}$-plane $\left(\checkmark\right.$ denotes the ${ }^{t} \mathrm{Bu}$ group).

\section{Surface Studies}

These days many efforts even from synthetic chemists are aimed at the preparation of functional nanostructures and more recently functional 2D, surface structures $(8 \mathrm{a}, 37)$. While the initial phase of the latter saw mainly attempts to prepare ordered porphyrin aggregates on surfaces the field has now moved to the logical construction of covalently linked systems and the identification and conformational investigation of individual molecules $(8 \mathrm{~b}, 37 \mathrm{a}, 38)$. Thus, the effects of the conformational flexibility of porphyrins observed in solution can also be traced in surface-porphyrin interactions. The many advances in this field now allow the preparation of defined structures on surfaces, e.g., porphyrin nanowires. Even dynamic processes, for example, the loss of axial ligands or metal-metal exchange between metalloporphyrins and metal surfaces can now be observed (39).

\section{How Nature Does It}

Yet, next to advances in solution and surface tuning in porphyrins, the question remains how a molecular fine-tuning of porphyrin cofactors is achieved in nature? Clearly, spatial control, i.e., a highly ordered arrangement of the cofactors plays a major role. The apoproteins (or self-aggregation in the chlorosomes) provide the structural framework for the $3 \mathrm{D}$ ordering and often modulate the porphyrin properties via axial coordination. Still, this alone cannot explain the ubiquitous and multifaceted roles of porphyrins in bioenergetics ranging from photosynthesis, methanogenesis, nitrite and sulfite reduction to vitamin $\mathrm{B}_{12}$ and heme chemistry in vivo. Very often the same chemical porphyrin cofactor (e.g., heme b, or chlorophyll) is used to promote chemically diverse reactions, ranging from oxidations and electron transfer to exciton transfer. A common denominator that links the molecular compound to its biological function is needed. We believe that the one fundamental property that can be used to approach this problem in a strategic manner is the conformational flexibility of porphyrins. Thus, both spatial and 
conformational control need to be considered to understand the biological fine-tuning of porphyrins in nature $(35,40)$.

As outlined in the section on macrocycle distortion, it is now well established that porphyrins are not necessarily simple, planar heteroaromatic compounds but rather can have distinctly different conformations, including very nonplanar ones. An expanding body of structural data for tetrapyrroles as isolated molecules and in proteins illustrates the considerable flexibility and the significant distortions that can be imposed on macrocycles by crystal packing, steric effects, or protein constraints (35).

By now evidence points towards a conformational control of the biological function in nature, too. Many regulatory phenomena in biological systems depend on axial coordination occurrences and a critical factor for all biological functions is the close interplay between bound cofactors and the respective apoprotein. Isolated pigments show physicochemical properties quite distinct from those in intact pigment-protein complexes (e.g., absorption maxima, redox potentials) and many individual cases of conformational effects in natural porphyrin-protein complexes have been identified (40). Thus, it is clear that the protein scaffold exerts conformational control on the porphyrin macrocycle.

It was less obvious whether these are individual cases or whether conformational control is a general concept. In order to address this question - for the specific case of the chlorophylls - we have recently performed an analysis of the available protein structural data for the bacteriochlorophylls in the photosynthetic reaction centers. The analysis gave statistically reliable evidence of the hypothesis that the protein induced cofactor conformation is a modulator of the bio-molecular function of each reaction center. Thus, the model shown in Figure 5 can serve as a general model to illustrate conformational control of tetrapyrrole cofactors in other proteins.

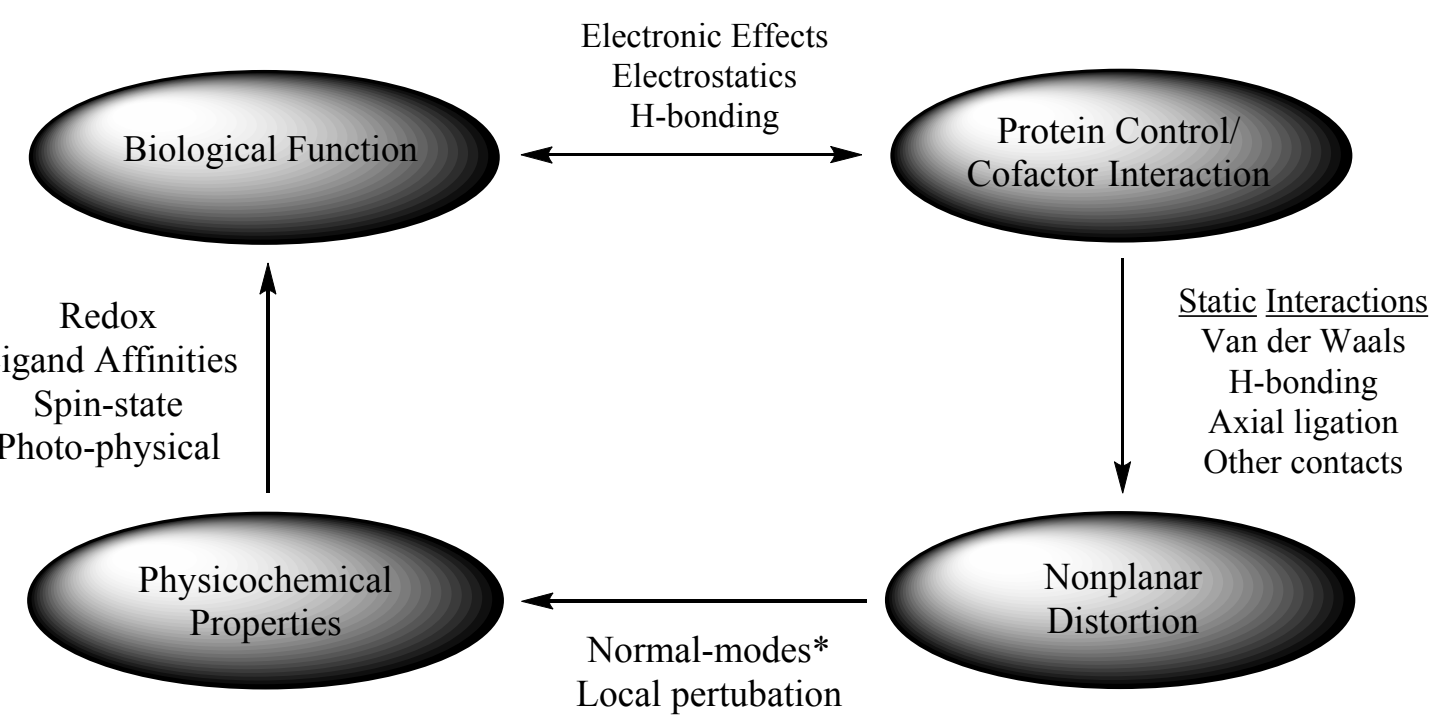

Figure 5. Illustration of the interrelationship between macrocycle conformation and biological function in conformational control.

Nature relies less on "chemical effects", i.e. the use of different cofactors, than thought - or than synthetic chemists do in the laboratory. Rather the same porphyrin cofactor is used for different chemical reactions and then modulated for its specific purpose through axial ligation, spatial control, and conformational modulation. The 
further analysis of the interrelationship between conformation and function will have implications for a wide range of different biological processes and for the efforts now devoted to biomimetic solar energy conversion, catalysis, cancer therapy, as well as basic mechanisms of electron transfer.

\section{Acknowledgments}

Our recent work in this area was supported by grants from Science Foundation Ireland (SFI P.I. 09/IN.1/B2650 and 12/TIDA/B2381).

\section{References}

1. K. M. Kadish, K. M. Smith, R. Guilard, Editors, Handbook of Porphyrin Science, Vol 1-10, World Scientific Publishing, Singapore (2010).

2. M. O. Senge, Chem. Commun., 47, 1943 (2011).

3. (a) K. S. Suslick, N. A. Rakow, M. E. Kosal, J. H. Chou, J. Porphyrins Phthalocyanines, 4, 407 (2000); (b) M. O. Senge, M. Fazekas, E. G. A. Notaras, W. J. Blau, M. Zawadzka, O. B. Locos and E. M. Ni Mhuircheartaigh, Adv. Mater., 19, 2737 (2007); (c) M. Zawadzka, J. Wang, W. J. Blau and M. O. Senge, Photochem. Photobiol. Sci., 12, 996 (2013).

4. (a) B. A. Gregg, M. A. Fox, A. J. Bard, J. Phys. Chem, 94, 1586 (1990); (b) A. Yella, H. W. Lee, H. N. Tsao, C. Y. Yi, A. K. Chandiran, M. K. Nareeruddin, E. W. G. Diau, C. Y. Yeh, S. M. Zakeeruddin, M. Grätzel, Science, 334, 629 (2011).

5. (a) R. R. Allison, G. H. Downie, R. Cuenca, X. H. Hu, C. J. H. Childs, C. H. Sibata, Photodiagn. Photodyn. Ther., 1, 27 (2004); (b) G. Jori, J. Photochem. Photobiol. B:Biol., 36, 87 (1996); (c) E. Pazko, C. Ehrhardt, M. O. Senge, D. P. Kelleher and J. V. Reynolds, Photodiagn. Photodyn. Ther., 8, 14 (2011); (d) G. M. F. G. Vaz, A. M. Davies, E. Paszko and M. O. Senge, PLOS One, 8, e70653 (2013).

6. L. R. Milgrom, The Colours of Life, Oxford University Press, Oxford (1997).

7. (a) D. Gust, T. A. Moore and A. L. Moore, Acc. Chem. Res., 34, 40 (2001); (b) D. M. Guldi, Chem. Soc. Rev., 31, 22 (2002) (c) J. Seth, V. Palaniappan, T. E. Johnson, S. Prathpan, J. S. Lindsey, D. F. Bocian, J. Am. Chem. Soc., 116, 10578 (1994).

8. (a) J. A. A. W. Elemans, R. van Hameren, R. J. M. Nolte and A. E. Rowan, Adv. Mater., 18, 1251 (2006); (b) A. A. Ryan, A. A. Cafolla and M. O. Senge, ECS Trans., 45, 77 (2013).

9. (a) A. K. Burrell, D. L. Officer, P. G. Plieger, Chem. Rev. 101, 2751 (2001); (b) J. S. Lindsey, Acc. Chem. Res., 43, 300 (2010); (c) M. O. Senge, Acc. Chem. Res., 38, 733 (2005); (d) M. G. H. Vicente, K. M. Smith, Curr. Org. Synth., 11, 3 (2014).

10. (a) M. O. Senge, Y. M. Shaker, M. Pintea, C. Ryppa, S. S. Hatscher, A. Ryan and Y. Sergeeva, Eur. J. Org. Chem., 237 (2010); (b) S. Plunkett and M. O. Senge, ECS Trans., 35, 147 (2011).

11. N. N. Servgeeva, M. O. Senge and A. Ryan, in Handbook of Porphyrin Science, K. M. Kadish, K. M. Smith, R.Guilard, Eds., Vol. 3, pp. 325-365, World Scientific Publishing, Singapore (2010). 
12. (a) M. O. Senge and N. N. Sergeeva, Angew. Chem. Int. Ed., 45, 2492 (2006); (b) M. O. Senge, I. Bischoff, Heterocycles, 65, 879 (2005); (c) N. N. Sergeeva and M. O. Senge, Tetrahedron Lett., 47, 6169 (2006); (d) I. Bischoff, X. Feng and M. O. Senge, Tetrahedron, 57, 5573 (2001).

13. S. M. LeCours, H.-W. Guan, S. G. DiMagno, C. H. Wang, and M. J. Therien, J. Am. Chem. Soc., 118, 1497 (1996).

14. (a) E. G. A. Notaras, M. Fazekas, J. J. Doyle, W. J. Blau and M. O. Senge, Chem. Commun., 21, 2166 (2007); (b) M. Zawadzka, J. Wang, W. J. Blau and M. O. Senge, J. Phys. Chem. A., 117(1), 15 (2013); (c) M. Zawadzka, J. Wang, W. J. Blau and M. O. Senge, Photochem. Photobiol. Sci., 12, 1811 (2013).

15. (a) A. C. Grimsdale, K. L. Chan, R. E. Martin, P. G. Jokisz and A. B. Holmes, Chem. Rev., 109, 897 (2009); (b) C. Borek, K. Hanson, P. I. Djurovich, M. E. Thompson, K. Aznavour, R. Bau, Y. Sun, S. R. Forrest, J. Brooks, L. Michalski and J. Brown, Angew. Chem. Int. Ed., 46, 1109 (2007).

16. (a) M. Balaz, H. A. Collins, E. Dahlstedt and H. L. Anderson, Org. Biomol. Chem., 7, 874 (2009); (b) K. Ogawa and Y. Kobuke, Anti-Cancer Agents Med. Chem., 8, 269 (2008); (c) K. Ogawa and Y. Kobuke, Org. Biomol. Chem., 7, 2241 (2009).

17. M.O. Senge and J.C. Brandt, Photochem. Photobiol., 87, 1240 (2011).

18. M. O. Senge and X. Feng, Tetrahedron Lett., 40, 4165 (1999).

19. Q. Ouyang, Y.-Z. Zhu, C.-H. Zhang, K.-Q. Yan, Y.-C. Li and J.-Y. Zheng, Org. Lett., 11, 5266 (2009).

20. A. A. Ryan and M. O. Senge, Eur. J. Org. Chem., 3700 (2013).

21. (a) A. G. Hyslop, M. A. Kellett, P. M. Iovine and M. J. Therien, J. Am. Chem. Soc., 120, 12676 (1998); (b) E. M. Finnigan, R. Rein, N. Solladié, K. Dahms, D. C. G. Götz, G. Bringmann and M. O. Senge, Tetrahedron, 67, 1126 (2011); (c) S. Punidha, N. Agarwal, I. Gupta and M. Ravikanth, Eur. J. Org. Chem., 1168 (2007).

22. K.-i. Sugiura, T. Matsumoto, S. Ohkouchi, Y. Naitoh, T. Ka-wai, Y. Takai, K. Ushiroda and Y. Sakata, Chem. Commun., 1957 (1999).

23. A. Tsuda and A. Osuka, Science, 293, 79 (2001).

24. N. Aratani and A. Osuka, Chem. Rec., 3, 225 (2003).

25. M. O. Senge, M. Pintea and A. Ryan, Z. Naturforsch. B, 66, 553 (2011).

26. L. He, Y.-Z. Zhu, J.-Y. Zheng, Y.-F. Ma and Y.-S. Chen, J. Photochem. Photobiol. A: Chem., 216, 15 (2010).

27. R. H. Felton and H. Linschitz, J. Am. Chem. Soc., 88, 1113 (1966).

28. N. S. Hush, J. R. Reimers, L. E. Hall, L. A. Johnston and M. J. Crossley, Ann. N.Y. Acad. Sci., 852, 1 (1998).

29. (a) T. L. Poulos, Chem. Rev., 114, 3919 (2014); (b) J. Deisenhofer, O. Epp, K. Miki, R. Huber and H. Michel, Nature, 318, 618 (1985); (c) G. McDermott, S. M. Prince, A. A. Freer, A. M. Hawthornthwaite-Lawless, M. Z. Papiz, R. J. Cogdell and N. W. Isaacs, Nature, 374, 517 (1995).

30. (a) H. L. Anderson, Inorg. Chem., 33, 972 (1994); (b) M. O. Senge, W. W. Kalisch and K. Ruhlandt-Senge, Chem. Commun., 2149 (1996); (c) S. Fukuzumi and T. Kojima, J. Mater. Chem., 181427 (2008).

31. A. A. Ryan, S. Plunkett, A. Casey, T. McCabe and M. O. Senge, Chem. Commun., 50, 353 (2014).

32. (a) J. E. Hutchison, T. A. Postlethwaite and R. W. Murray, Langmuir, 9, 3277 (1999); (b) K. Shimazu, M. Takechi, H. Fujii, M. Suzuki, H. Saiki, T. Yoshimura 
and K. Uosaki, Thin Solid Films, 273, 250 (1996); (c) C. Clausen, D. T. Gryko, R. B. Dabke, N. Dontha, D. F. Bocian, W. G. Kuhr and J. S. Lindsay, J. Org, Chem., 65, 7371 (2000).

33. A. R. Katritzky, G. R. Khan and O. A. Schwarz, Tetrahedron Lett., 25, 1223 (1984).

34. (a) T. Itoh and T. Mase, Org. Lett., 6, 4587 (2004); (b) T. Itoh and T. Mase, J. Org. Chem., 71, 2203 (2006).

35. (a) M. O. Senge, in The Porphyrin Handbook, K. M. Smith, K. M. Kadish and R. Guilard, Editors, Vol. 1, pp. 239, CRC Press, Boca Raton, USA (2000); (b) M. O. Senge, Chem. Commun., 243 (2006).

36. (a) M. O.Senge, J. Richter, I. Bischoff and A. Ryan, Tetrahedron, 66, 3508 (2010); (b) M. O. Senge, Acta Crystallogr., C67, m39 (2010); (c) B. Röder, M. Büchner, I. Rückmann and M. O. Senge, Photochem. Photobiol. Sci., 9, 1152 (2010); (d) M. Zawadzka, J. Wang, W. J. Blau and M. O. Senge, J. Porphyrins Phthalocyanines, 17, 1129 (2013); (e) C. Ryppa, M. O. Senge, S. S. Hatscher, E. Kleinpeter, P. Wacker, U. Schilde and A. Wiehe, Chem. Eur. J., 11, 3427 (2005).

37. (a) L. Grill, M. Dyer, L. Lafferentz, M. Persson, M. V. Peters and S. Hecht, Nature Nanotechn., 2, 687 (2007); (b) F. D’Souza and O. Ito, Chem. Soc. Rev., 41, 86 (2007).

38. (a) J. K. Gimzewski, T. A. Jung, M. T. Cuberes and R. R. Schlitter, Surf. Sci., 386, 101 (1997); (b) T. A. Jung, R. R. Schlitter and J.K. Gimzewski, Nature, 386, 696 (1997); (c) S. A. Krasnikov, C. M. Doyle, N. N. Sergeeva, A. B. Preobrajenski, N. A. Vinogradoc, Y. N. Sergeeva, A. A. Zakharov, M. O. Senge and A. A. Cafolla, Nano Res., 4, 376 (2011).

39. (a) J. P. Beggan, S. A. Krasnikov, N. N. Sergeeva, M. O. Senge and A. A. Cafolla, Nanotechnology, 23, 235606 (2012); (b) C. M. Doyle, J. P. Cuniffe, S. A. Krasnikov, A. B. Preobrajenski, Z. S. Li, N. N. Sergeeva, M. O. Senge and A. A. Cafolla, Chem. Commun., 50, 3447 (2014).

40. (a) R. Huber, Eur. J. Biochem., 187, 283 (1990); (b) M. O. Senge, J. Photochem. Photobiol. B:Biol., 16, 3 (1992); (c) J. A. Shelnutt, X. Z. Song, J. G. Ma, S. L. Jia, W. Jentzen and C. J. Medforth, Chem. Soc. Rev., 27, 31 (1998). 